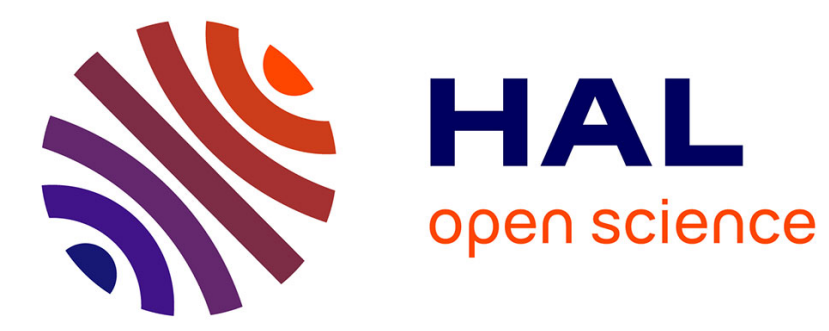

\title{
L'Albanie aux marges de l'Europe
}

Gilles de Rapper

\section{To cite this version:}

Gilles de Rapper. L'Albanie aux marges de l'Europe. Boris Petric \& Jean-François Gossiaux. Europa mon amour. 1989-2009: un rêve blessé, Autrement, pp.98-109, 2009. halshs-00400343v2

\section{HAL Id: halshs-00400343 \\ https://shs.hal.science/halshs-00400343v2}

Submitted on 14 Sep 2009

HAL is a multi-disciplinary open access archive for the deposit and dissemination of scientific research documents, whether they are published or not. The documents may come from teaching and research institutions in France or abroad, or from public or private research centers.
L'archive ouverte pluridisciplinaire HAL, est destinée au dépôt et à la diffusion de documents scientifiques de niveau recherche, publiés ou non, émanant des établissements d'enseignement et de recherche français ou étrangers, des laboratoires publics ou privés. 
Article paru dans J.-F. Gossiaux et B. Petric (éds.), Europa mon amour. 1989-2009 : un rêve blessé. Paris, Éditions Autrement (2009), p. 98-109.

\title{
L'ALBANIE AUX MARGES DE L'EUROPE
}

\author{
Gilles de Rapper
}

Lorsque j’ai visité l'Albanie pour la première fois, en 1994, le Parti démocratique était au pouvoir depuis deux ans, le "monisme», ainsi que beaucoup d'Albanais avaient pris l'habitude d'appeler l'ancien régime, appartenait au passé et les slogans promettaient de «faire l'Albanie comme l'Europe ${ }^{1} »$. Pourtant, le chemin paraissait encore long et [100] l'arrivée en Albanie représentait un dépaysement total pour l'étranger que j'étais. J'arrivai depuis la Grèce et entrai par le poste frontière de Kakavijë, dans l'extrême sud albanais. L'entrée sur le territoire albanais se faisait par une grille fermant la route et que les gardesfrontières grecs ouvraient au coup par coup. De l'autre côté, comme derrière les barreaux d'une prison, une masse d'hommes, de femmes et d'enfants attendaient de pouvoir entrer en Grèce en brandissant leurs passeports vers les policiers grecs. C'est un peu avant cette barrière que je rencontrai pour la première fois Ismail, un Albanais d'une quarantaine d'années. Chauffeur de taxi, il était autorisé par les gardes-frontières, avec quelques collègues, à attendre les clients du côté grec de la frontière. Nous sommes devenus amis, il m’a invité dans sa famille. Je suis retourné chez lui à deux reprises en 1995, puis nous nous sommes perdus de vue jusqu'en 2007. Entre temps, l'Albanie avait connu la guerre civile de 1997, l'arrivée des réfugiés kosovars et une forme d'alternance politique et, comme cela est naturel, la situation d'Ismail et de sa famille avait changé2.

\footnotetext{
1 En décembre 1990, une révolte étudiante conduit à un soulèvement de la population de la capitale : le régime accepte le principe du pluripartisme et le Parti démocratique, premier parti d'opposition, est créé. Des élections sont organisées en mars 1991, remportées par le Parti du travail, grâce au vote des campagnes. Le mécontentement envers l'ancien système s'accroît cependant et conduit le gouvernement de stabilisation instauré en juin 1991 à organiser de nouvelles élections en mars 1992. Elles sont remportées par le Parti démocratique dont le chef, Sali Berisha, devient président de la république.

2 En 1997, les systèmes d'épargne pyramidale qui fleurissaient depuis quelques années s'effondrèrent, ruinant des centaines de milliers d'épargnants et plongeant l'Albanie dans le chaos. De 1997 à 2005, le pays fut dirigé par le Parti socialiste (successeur du Parti du travail), qui dut rétablir l'autorité de l'État, réorganiser
} 
Ismail habitait à Memaliaj, une petite ville du Sud de l'Albanie située sur la route qui mène depuis Tirana vers la frontière grecque. Il vivait avec sa femme, Drita, employée dans la station électrique de la ville, et ses deux enfants, un garçon et une fille âgés de 16 et 13 ans. Memaliaj est une ville minière et la fermeture progressive de la mine au début des années 1990 avait laissé de nombreuses familles sans ressources. Chaque matin, Ismail partait avant l'aube pour attendre à la frontière, à 70 kilomètres de chez lui, l'arrivée des autocars en provenance d'Athènes. Il conduisait ensuite les "réfugiés ", ainsi que l'on désignait et que se désignaient ceux qui partaient travailler en Grèce, de retour chez eux dans les diverses régions du Sud et parfois jusqu'à Tirana. Comme de nombreux détenteurs du permis de conduire, une denrée rare jusqu'en 1991, il s'était établi comme chauffeur de taxi privé et profitait de la désorganisation et de la lenteur des transports en commun pour offrir ses services sur le principal axe routier reliant Tirana à la Grèce. Lui-même n'envisageait pas de partir en Grèce, comme le [101] faisaient beaucoup de ses voisins, que la fermeture de la mine avait condamnés au chômage. Sa femme, Drita, avait conservé son emploi et son salaire, même faible, était une rentrée d'argent assurée.

En 2007, j’ai retrouvé Ismail et Drita dans le même appartement, refait à neuf. Drita travaille toujours à la station électrique tandis qu'Ismail a abandonné l'activité de chauffeur de taxi pour travailler lui aussi, en tant que chauffeur, à la compagnie d'électricité. En 1995 déjà, il se plaignait de la concurrence que se livraient les chauffeurs de taxi sur la route de la frontière et les troubles de 1997 l'ont décidé à arrêter cette activité. Edi, le fils aîné, travaille à Tirana. Après avoir terminé le lycée, il a d'abord fait quatre années de droit à l'université puis, ne trouvant pas de travail, quatre années d'économie. Il s'est marié à l'été 2008 avec une fille de Memaliaj. Elona, la cadette, vit à Rhodes, où elle a rejoint le frère et la sœur d'Ismail. Elle travaille dans une boulangerie et envisage de se marier avec un Grec; en novembre 2007, ses parents sont allés à Rhodes pour assister à son baptême : le fiancé et sa famille ne pouvaient envisager un mariage avec une musulmane et Elona a dû se convertir. Ismail et Drita, qui se disent musulmans, ont accepté : leur fille est devenue très croyante depuis qu'elle vit en Grèce et ils ne veulent pas s'opposer à ce qu'elle désire et puis, comme on l'entend souvent dans un pays où coexistent musulmans et chrétiens, « finalement, il n'y a qu'un seul dieu ».

\section{Une société en mouvement}

Si Ismail et Drita n'ont pas bougé, l'itinéraire de leurs enfants illustre un phénomène majeur de la société albanaise d'après 1990 : celui de la libéralisation des déplacements, à l'intérieur comme à l'extérieur du pays. Celle-ci a d'emblée pris une ampleur remarquable et les images de l'«exode» de 1991, lorsque des bateaux bondés de réfugiés albanais traversaient l'Adriatique pour accoster dans les ports italiens, ont marqué les opinions publiques occidentales. Moins médiatisées, les traversées de la frontière terrestre avec la

l'économie et faire face à l'arrivée des réfugiés fuyant le Kosovo en guerre (1999). Le Parti démocratique revient au pouvoir en 2005 et Sali Berisha devient Premier ministre. 
Grèce ont pourtant rapidement dépassé le nombre de départs vers l'Italie. Près de vingt ans plus tard, les Albanais continuent de franchir les frontières de leur pays ; l'émigration touche toute la population, par ses effets démographiques (la population de l'Albanie est passée de 3250000 habitants en 1990 à 3069275 en 2001; 500000 Albanais vivraient en Grèce) comme par les revenus qu'elle génère pour ceux qui ne sont pas partis.

[102] Situé sur la route de la Grèce, Memaliaj est touché de deux manières par ces mouvements migratoires. D’un côté, de nombreuses familles vivent en Grèce et ne reviennent qu'occasionnellement en ville (en avril 2008, sur les quinze appartements de l'immeuble d'Ismail, cinq étaient inoccupés en raison du départ des familles vers la Grèce). De l'autre, le passage des cars de migrants génère une activité économique qui profite aux propriétaires de restaurants ou de stations-service. Un matin d'avril 2008, Ismail nous emmène ainsi au Paris, un café-restaurant situé au bord de la route nationale et ouvert deux ans plus tôt par une nièce de Drita et son mari, qui sont aussi propriétaires de la stationservice de l'autre côté de la route. Un autocar de la ligne Tirana-Athènes est garé devant l'établissement. Ses occupants sont attablés à l'intérieur et prennent un petit-déjeuner de riz et de soupe de tripes. Trois hommes remplissent des papiers à une table voisine. La serveuse leur apporte ensuite des assiettes de viande rôtie, un plat de feuilleté (byrek) et des bouteilles d'eau minérale. "Ce sont les chauffeurs du car, explique Ismail, ils sont nourris gratuitement. En échange, ils font toujours halte ici et amènent des clients. »

Les mouvements ne se font pas que vers l'extérieur du pays. Ils concernent aussi les relations entre les campagnes et les villes. Le démantèlement des coopératives agricoles et l'incertitude sur la propriété de la terre s'ajoutent à l'image d'une vie plus facile et moderne en ville pour inciter de nombreuses familles rurales à s'installer dans les centres urbains régionaux ou à Tirana.

Au mouvement des hommes vers l'étranger répond l'arrivée de nombreux biens de consommation depuis l'extérieur. Au début des années 1990, toutes les familles, quel que fût leur niveau de revenu, avaient pour objectif prioritaire l'équipement de leur maison : les téléviseurs couleur et les réfrigérateurs étaient encore rares et réservés à une élite privilégiée. Ismail s'est ainsi d'abord procuré un téléviseur avec télécommande; puis sont venus le réfrigérateur, installé dans le vestibule, et la cuisinière électrique remplaçant le poêle à bois. Au printemps 1995, Ismail a fait le voyage jusqu'à Tirana pour rapporter un chauffe-eau électrique (de fabrication italienne) et, quelques mois plus tard, la famille possédait aussi un lave-linge. L'appartement avait encore changé lors de mon retour en novembre 2007 : la cuisine et la salle de bains ont été entièrement refaites, le réfrigérateur a quitté le vestibule pour s'intégrer dans les nouveaux éléments de cuisine à côté du four à micro-ondes, les toilettes allaturka ont été remplacées par une cuvette [103] allafranga et une baignoire a fait son apparition. Dans le salon, les vieux divans que l'on retrouvait uniformément dans tous les intérieurs ont fait place à de volumineux fauteuils et canapés convertibles; le sol a été carrelé et, sur la table basse, deux poissons rouges tournent dans un bocal. En rentrant du travail, Drita et Ismail se changent et revêtent un ensemble de jogging, une habitude qui leur était inconnue au milieu des années 1990 et qui s'est répandue en Albanie par l'intermédiaire des migrants revenant de Grèce. 
L'accent mis sur la libéralisation du mouvement depuis 1990 ne doit pas faire oublier que la population albanaise n'est pas restée figée pendant la période précédente. Sans remonter jusqu'à l'émigration d'avant la Seconde Guerre mondiale qui, depuis la fin du XIX ${ }^{\mathrm{e}}$ siècle, avait vu de nombreux Albanais partir pour les centres urbains de l'Empire ottoman et, plus tard, vers ceux d'Amérique ou d'Australie, il faut rappeler qu'au lendemain de la guerre la situation dans les campagnes les plus durement touchées par les combats - contre les occupants allemands ou entre factions rivales - poussait une partie de la population à se diriger vers les villes. Un peu plus tard, la politique d'industrialisation du pays menée à partir des années 1950 exigea une main-d'œuvre ouvrière qui n'existait guère avant le conflit et qui fut créée grâce aux arrivées depuis les campagnes. La ville de Memaliaj ellemême date de ces années, lorsqu'il fallut loger les ouvriers de la mine de charbon, dont le nombre finit par atteindre 3000 dans les années 1980. Les immeubles les plus anciens, comme celui dans lequel habitaient les parents d'Ismail en 1995, datent de 1954. Celui qu'occupent Ismail et Drita, haut de quatre étages, appartient à la dernière phase, celle de 1988. La ville, avec sa place centrale fermée par le Palais de la culture, son monument commémoratif des combats de la Seconde Guerre mondiale et ses rangées d'immeubles, fut bâtie en plaine, entre la rive du fleuve et une colline sur laquelle se tient depuis beaucoup plus longtemps le village de Memaliaj. Les ouvriers de la mine furent recrutés dans les villages environnants, comme le fut le père d'Ismail, tandis que les ingénieurs et les cadres venaient de plus loin et apportaient avec eux d'autres habitudes et d'autres coutumes. La population de la ville s'en trouve beaucoup plus mêlée que celle des centres urbains plus anciens et que celle des campagnes. Cette mixité géographique et sociale est aussi une mixité religieuse : Drita raconte qu'il y a beaucoup d'«infidèles» (kaur) à Memaliaj, des chrétiens orthodoxes originaires du Dropull, une région habitée par la minorité grecque d'Albanie. Ils sont [104] arrivés à l'époque de la mine et sont restés. Elle cite ses voisins du dessous, des chrétiens dont les enfants ont épousé des musulmans, et le propre frère d'Ismail, qui a épousé une chrétienne. Depuis la fin de la dictature et le rétablissement de la liberté religieuse, cette mixité est parfois source de tensions. Les terres sur lesquelles la ville de Memaliaj a été bâtie appartenaient à la communauté religieuse des bektachis, un ordre hétérodoxe musulman bien implanté en Albanie méridionale. Son représentant local, le $b a b a$, qui veille sur le lieu de culte situé dans le vieux village, déplore d'autant plus la perte de ces terres qui, louées aux éleveurs ou aux agriculteurs, étaient une source de revenus pour la communauté, que le renouveau religieux des années 1990 s'est traduit par la construction d'une grande église orthodoxe en bordure de la ville, démesurée par rapport au nombre de fidèles, même si les espoirs de migration vers la Grèce chrétienne incitent des musulmans à se déclarer chrétiens et à fréquenter les églises.

Le caractère massif de la migration des années 1990 et 2000 ne doit pas masquer le fait que la mobilité n'est pas permise à tous de la même manière et qu'elle n'a pas connu les mêmes formes tout au long de cette période. En 1995, ni Ismail ni sa proche famille n'avaient fait l'expérience de la migration vers la Grèce. Ses revenus en tant que chauffeur de taxi, joints à la sécurité de l'emploi dont jouissait Drita en tant qu'employée de la compagnie d'électricité, leur permettaient d'envisager l'éducation des enfants et l'aménagement de l'appartement sans le recours à l'émigration. L'année 1997 a marqué une 
rupture dans la vie des familles qui avaient su s'adapter au changement de régime quelques années plus tôt. À la désorganisation de l'État, à l'arrêt de l'activité économique et au départ des investisseurs étrangers s'est ajoutée l'insécurité due à la formation de bandes armées et au climat de guerre civile. Memaliaj fut à cet égard fortement touchée. Les dépôts d'armes, nombreux et bien fournis dans un pays où toute la population, femmes et enfants compris, était soumise à des périodes d'entraînement militaire plusieurs fois par an, avaient été ouverts et tout le monde s'était servi. Ce fut une "vraie guerre ", raconte Ismail, de mars à juin 1997. Un jour, treize garçons de la ville ont été tués, « soi-disant par des Tsiganes mais sans doute par les forces gouvernementales »; devant la station électrique où travaille Drita, un petit monument rappelle la mort d'une mère et de son bébé, tués accidentellement par un jeune garçon qui jouait avec une kalachnikov.

À la suite de ces événements, le frère et la sœur d'Ismail quittèrent l'Albanie pour la Grèce et finirent par s'établir à Rhodes, où la fille [105] d'Ismail les a rejoints à la fin de sa scolarité obligatoire. Après la mort du père, en 2004, ils firent aussi venir leur mère. Drita s'ennuie de sa fille, mais n'envisage pas de la rejoindre en Grèce : «Je suis aussi bien ici, ditelle en novembre 2007, qu'à faire des ménages en Grèce. » Ismail dit que s'il le pouvait il s'installerait bien à Rhodes, mais à plus de cinquante ans et sans connaissance du grec, il a peu de chances d'y trouver du travail et risque de s'y ennuyer. Il évoque sa mère qui vit làbas, mais qui n'a pas appris la langue, ne sort jamais et ne voit personne en dehors de la famille. Quelques mois plus tard, en avril 2008, la mère rentre définitivement en Albanie : après quatre ans passés à Rhodes, elle a décidé de revenir à Memaliaj, où elle a "ses voisines et ses proches ». Ismail et Drita admettent aussi que s'ils ne bougent pas, c'est que leurs revenus ne le leur permettent pas. Avec un salaire mensuel de 250 euros chacun, ils s'en sortent parce qu'ils vivent dans une petite ville, où la vie est moins chère qu'à Tirana ; ils ne pourraient pas vivre dans les mêmes conditions dans la capitale.

\section{À la conquête de l'Europe}

Il n'en reste pas moins que l'ouverture des frontières est une réalité et qu'elle a profondément transformé la vie de tous les Albanais, qu'ils soient ou non partis en migration. En novembre 2007, Drita profite de ses moments de loisirs pour étudier une méthode de grec. Dans quelques jours, Ismail et elle ont rendez-vous au consulat grec de Gjirokastër pour recevoir la réponse de leur demande de visa pour la Grèce où ils vont assister au baptême de leur fille. «La première fois où nous avons pris l'avion pour aller à Rhodes, raconte-t-elle, nous étions complètement perdus, nous ne pouvions rien dire, nous nous perdions dans l'aéroport d'Athènes sans pouvoir demander notre chemin. » L'apprentissage et la pratique du grec - comme de la plupart des langues étrangères à l'exception du russe et du français - étaient interdits pendant la dictature, même dans le Sud où les contacts avec les membres de la minorité grecque d'Albanie étaient plus fréquents. Les voyages à l'étranger étaient impossibles, tandis que Drita et Ismail feuillettent aujourd'hui des albums photographiques les montrant en vacances dans les sites touristiques de Rhodes. 
Le contact avec le monde extérieur a commencé pour eux, comme pour beaucoup d'Albanais à la fin des années 1980 et au début des [106] années 1990, par la télévision. Avant même la chute de la dictature, qui interdisait les chaînes étrangères, les Albanais étaient devenus maitres dans l'installation d'antennes de fortune permettant de capter les chaînes italiennes, grecques ou yougoslaves dont l'émission couvrait une partie du territoire albanais. C'est ainsi qu'en 1994, les enfants d'Ismail comprenaient couramment l'italien sans avoir suivi de cours et sans n'avoir jamais visité l'Italie. Les télévisions étrangères, et les chaînes italiennes en particulier, fournissaient ainsi un flot d'images sur l'Europe et le monde de l'Ouest qui risquaient d'être mal comprises ou mal interprétées par des spectateurs peu au fait des réalités. Au cours de mon séjour d'octobre 1995, les deux enfants (l'aîné avait alors seize ans) n'auraient jamais manqué un épisode de la série américaine Baywatch (Alerte à Malibu) diffusée par une chaîne privée italienne. Les adultes prétendaient en général ne pas regarder et disaient ne pas comprendre l'italien, mais beaucoup regardaient (ce que dit Pamela Anderson n'a pas besoin d'être compris), fascinés par le spectacle d'un monde où tout le monde est riche et beau et où tout finit par s'arranger. «Oh là là !» me dit un jour Drita, alors que Mitch venait de sauver deux jolies baigneuses de la noyade, «ces Italiens, quels gens! Et quel pays!». Son fils la corrigea : «Ce n'est pas l'Italie, c'est l'Amérique. » «L'Amérique ? » reprit-elle, incrédule, « et depuis quand les Américains parlent-ils italien ?» «Ils parlent italien parce que le film est doublé, mais ça se passe en Amérique, en Californie.» «Oh», fit Drita en se tournant vers moi, avec un geste de la main comme pour effacer la télévision, "l'Amérique, l'Italie, la Californie, quelle importance ! Mais pauvres de nous, où sommes-nous ?»

Treize ans plus tard, le téléviseur après avoir quitté le salon, qui servait jusqu'à leur départ de la maison de chambre aux enfants, pour passer dans la chambre à coucher, est de nouveau dans le salon et toujours allumé. Pour 500 leks par mois (4 euros), Ismail et Drita sont abonnés au câble et reçoivent des centaines de chaînes albanaises et étrangères. Chaque soir, Drita suit une telenovela allemande doublée en italien et sous-titrée en albanais, en prenant soin de préparer le souper avant le début de son feuilleton, mais, en ce printemps 2008, comme dans de très nombreux foyers albanais, en ville comme à la campagne, l'émission à ne pas rater est la première édition de Big Brother Albania, diffusée par une chaîne privée et produite par une société italienne. Pendant six mois, une dizaine de jeunes vivent dans un immense appartement, privés de tout contact avec le monde extérieur et filmés en [107] permanence par de multiples caméras. Chaque semaine, les téléspectateurs sont invités à voter pour le départ ou le maintien des uns et des autres. L'Albanie est enfin comme le reste de l'Europe.

\section{Avant, après}

Dix-sept ans après la chute de la dictature, l'existence des familles comme celles d'Ismail reste prise entre un passé que l'on considère comme définitivement terminé, mais que l'on n'oublie pas, et un présent fait d'improvisation et de découverte d'un monde dont les règles sont perçues comme imposées depuis l'extérieur. La société albanaise d'aujourd'hui demeure profondément marquée par des lignes de fracture héritées de la 
période communiste. C'est le cas de la vie politique et de la lutte que se mènent, depuis l'autorisation du pluripartisme en décembre 1990, le Parti démocratique et le Parti socialiste. La mairie de Memaliaj est passée au Parti démocratique lors des élections locales de 2007, tout comme les villages environnants, après avoir longtemps été tenue par le Parti socialiste. Ismail explique ce changement par des «affaires» dans lesquelles les élus socialistes se sont retrouvés impliqués et qui ont incité les électeurs à voter pour leurs opposants. Les travaux effectués en 2005 pour améliorer les conditions de vie des habitants de Memaliaj (asphaltage et éclairage des rues, installation du tout-à-l'égout) n'ont pas suffi à la mairie pour garder le soutien de la population. D'une manière générale, le retour au pouvoir du Parti démocratique en 2005 au niveau national fut la conséquence de la mauvaise réputation du Parti socialiste après huit années de pouvoir sans partage sur le pays : corruption, népotisme, abus de bien sociaux et implication personnelle des élus et de ministres dans des activités économiques fort profitables sont des accusations qui furent largement portées contre le Parti socialiste. Ismail, comme en 1995, continue de se dire socialiste. Non pas par attachement à l'ancien régime, mais par sentiment d'appartenance à ce qu'il appelle la "classe ouvrière », sentiment qu'il explique par le fait que son père a travaillé pendant des années à la mine. Outre les affaires de corruption et de malversations, les accusations que l'on porte contre le parti au pouvoir tournent autour du respect des libertés, un acquis de la "démocratie », terme par lequel on désigne la chute de la dictature. Accuser le gouvernement de porter atteinte aux libertés et de faire un usage immodéré de la répression revient à le renvoyer à des pratiques d'avant 1990 .

[108] La polarisation entre les deux forces politiques s'exprime aussi dans le recrutement des fonctionnaires et plus généralement dans le marché de l'emploi. Au niveau national comme au niveau local, un changement de direction politique s'accompagne systématiquement d'un renouvellement des effectifs des différentes administrations. Ismail se plaint ainsi que depuis le retour du Parti démocratique au pouvoir en 2005, la compagnie d'électricité a recruté des gens en raison de leur affiliation partisane. Ces nouveaux collègues, déplore-t-il, nommés pour des raisons politiques, se croient plus compétents que les autres, mais ne connaissent rien au travail.

Les références au passé d'avant 1a démocratie sont fréquentes lorsqu'il s'agit de parler des relations sociales dans l'Albanie d'aujourd'hui. Dans les conversations quotidiennes, l'insécurité actuelle est opposée à l'ordre et à la tranquillité qui régnaient pendant la dictature. Drita raconte que lorsqu'elle était jeune fille et étudiante à Gijirokastër, elle prenait le bus seule et personne ne l'importunait, ce qui selon elle ne serait plus possible aujourd'hui. Elle rappelle cependant incidemment que lorsqu'elle a terminé le lycée technique et qu'elle a été nommée dans un village de la région, son père a refusé de la laisser partir; elle a dû attendre un an avant d'être nommée à la station de Memaliaj et commencer à travailler. Les relations entre hommes et femmes sont souvent citées comme ayant profondément changé depuis la « fin du système ». Lorsque Drita décrit la situation de son fils à Tirana, qui cohabite depuis cinq ans avec sa future femme, sans être marié, Ismail intervient en disant que cela prouve qu'ils sont « devenus européens ». Ce que l'on oppose à ces nouveaux comportements est moins la morale communiste qu'un certain conservatisme que la dictature avait contribué à entretenir. Dans le bus qui part tôt le matin 
de Memaliaj pour Gijirokastër, des passagers âgés critiquent justement l'habitude nouvelle pour les couples de cohabiter plutôt que de se marier; ils déplorent de même la recrudescence des divorces et le fait que les femmes veulent être à égalité avec les hommes.

La comparaison tourne souvent autour des inégalités et de l'apparition de classes sociales dans l'Albanie post-communiste. Les disparités sont désormais flagrantes entre l'agglomération de Tirana, qui concentre la richesse et la modernité, et le reste du pays, où les transformations se font à un autre rythme; elles sont flagrantes entre les villes, qui voient leur population augmenter, et les villages qui se vident et ne conservent qu'une population âgée. Plus encore, l'adaptation rapide de certains individus aux nouvelles règles du jeu rend leur réussite suspecte aux [109] yeux de ceux qui sont restés attachés aux anciennes règles. Alors que je parle à Drita de l'essor du secteur de la construction et des immeubles qui poussent dans la ville de Gjirokastër, elle répond qu'il ne faut pas y voir un signe de bonne santé de l'économie et du pays : selon une opinion répandue, les gens qui peuvent construire de tels immeubles ont dû s'enrichir par la corruption et les trafics ; « ceux qui travaillent », c'est-à-dire à qui l'État verse un salaire, en seraient incapables. Dans les régions frontalières, l'émigration et les activités économiques liées à la frontière accentuent les disparités entre ceux qui partent et ceux qui restent, entre ceux qui participent à la nouvelle économie internationale et ceux qui subsistent sur les ressources locales.

Une après-midi d'avril, après la visite de la mine désaffectée où quelques villageois remplissent des sacs de charbon qu'ils iront vendre en ville, nous buvons un café avec un ami d'Ismail, là où la route de la mine rejoint la route nationale. La vue porte au loin dans la vallée et sur les montagnes environnantes; Berti, qui se dit sans emploi après quelques tentatives infructueuses de migration vers la Grèce, vante la beauté des paysages et l'intérêt de ce point d'observation d'où, dit-il, «on peut voir passer aussi bien une voiture à 200000 euros qu'un paysan sur son âne.» 